\title{
Integrated Power Flow and Short Circuit Calculation Method for Distribution Network with Inverter Based Distributed Generation
}

\author{
Shan Yang ${ }^{1}$ and Xiangqian Tong ${ }^{1,2}$ \\ ${ }^{1}$ School of Automation and Information Engineering, Xian University of Technology, Xian, Shaanxi 710048, China \\ ${ }^{2}$ Xian Hailian Petrochemical Technologies Co., Ltd., Xian, Shaanxi 710065, China
}

Correspondence should be addressed to Xiangqian Tong; lstong@mail.xaut.edu.cn

Received 27 January 2016; Revised 27 May 2016; Accepted 30 May 2016

Academic Editor: Yan-Wu Wang

Copyright (C) 2016 S. Yang and X. Tong. This is an open access article distributed under the Creative Commons Attribution License, which permits unrestricted use, distribution, and reproduction in any medium, provided the original work is properly cited.

\begin{abstract}
Power flow calculation and short circuit calculation are the basis of theoretical research for distribution network with inverter based distributed generation. The similarity of equivalent model for inverter based distributed generation during normal and fault conditions of distribution network and the differences between power flow and short circuit calculation are analyzed in this paper. Then an integrated power flow and short circuit calculation method for distribution network with inverter based distributed generation is proposed. The proposed method let the inverter based distributed generation be equivalent to $I \theta$ bus, which makes it suitable to calculate the power flow of distribution network with a current limited inverter based distributed generation. And the low voltage ride through capability of inverter based distributed generation can be considered as well in this paper. Finally, some tests of power flow and short circuit current calculation are performed on a 33-bus distribution network. The calculated results from the proposed method in this paper are contrasted with those by the traditional method and the simulation method, whose results have verified the effectiveness of the integrated method suggested in this paper.
\end{abstract}

\section{Introduction}

The reserved finiteness and destruction of environment by fossil energy have made the application of distributed generation (DG) widespread gradually [1]. The grid-connected DG in distribution network can reduce the loss of fossil energy and network lines and improve the utilization of energy and the reliability of customers. However, DG also brings some unfavorable effects at the same time. For example, in normal operation condition of distribution network, the electrical states of distribution network will be changed due to the random power output from DG. And the changed installed position and installed capacity of DG may bring some unfavorable effects on voltage and loss. In fault operation condition of distribution network, the magnitude and direction of short circuit current flowing through the line could be changed too, and the misoperation and failure action of traditional 3-sectional current protection could happen $[2,3]$. Power flow and short circuit calculation are the basis of analyzing the normal and fault conditions of distribution network, respectively. Therefore, it is important to study the power flow and short circuit calculation for research of distribution network with DG.

The DGs in distribution network are mainly divided into generator based DG and inverter based DG, of which the inverter based DG is applied in distribution network widely, such as photovoltaic (PV), permanent magnet synchronous generator (PMSG), and energy storage system (ESS). Therefore, it is significant to study the power flow and short circuit calculation methods for distribution network with inverter based DGs. As the research focus in this paper, the DG mentioned below without additional instruction referred to is expressed as inverter based DG.

Both in power flow and in short circuit calculation, the research at present mainly consists of building equivalent model of inverter based DG and putting forward corresponding calculation method. Being unlike generator based DG, the output characteristic of inverter based DG is mainly dominated by its control unit. In power flow calculation, the equivalent models of DGs under different control are 
different. In constant power control, the DG should be equivalent to $P Q$ bus while in constant voltage control, the DG should be equivalent to PV bus if the reactive power output from DG is within limit. If the reactive power output from DG is beyond the limit, the DG should be equivalent to $P Q$ bus $[4,5]$. Therefore, the equivalent model of inverter based DG is mainly modeled as PQ bus or PV bus. And for calculation method, back/forward sweep method and Newton method are generally used the same as traditional power flow calculation [6-8].

In short circuit calculation, the present fault equivalent models of inverter based DG have had the following three forms. (1) At the assumption of constant active and reactive power output from DG during a fault, DG should be equivalent to constant power model [9]. (2) According to the current limiting characteristic of inverter, DG should enter current limiting status during a fault. As a result, DG can be equivalent to constant current source approximately $[10,11]$. (3) The fault equivalent model of inverter based DG is decided by judging the current limiting status of DG. The DG entering the current limiting status is equivalent to constant current model. Conversely, the DG is equivalent to constant power model [12, 13]. As far as short circuit calculation method is concerned, the superposition theorem, which calculates the normal component and fault component of distribution network, respectively, and superposes them [14], is usually adopted.

From the researches introduced above, it can be seen that, in power flow calculation, the equivalent model of inverter based DG is processed ideally. The current limiting status of inverter based DG has not been considered. For example, DG may enter into the current limiting status when the voltage stability of distribution network is analyzed with power flow calculation for the loads increasing too much. In this case, the control objectives of inverter cannot be maintained. Hence, if the inverter based DG is still modeled as $P Q$ or PV bus, the impact of DG on power flow cannot be correctly included. Similarly, in short circuit calculation, DG may enter into the current limiting status as well for the decreased voltage at point of common coupling (PCC) during a fault. At this moment, the inverter based DG should be equivalent to current source, which has been mentioned in some researches already. But the current source models proposed in those researches are ideally for the simplified analysis of current output regulation. In addition, a lot of inverter based DGs have been configured with Low Voltage Ride Through (LVRT) control at present [15]. Therefore, the fault equivalent model of inverter based DG under LVRT control should be studied.

In this paper, the equivalent model of inverter based DG in normal and fault operation conditions of distribution network and the difference between power flow and short circuit calculation are analyzed at first. Then, an integrated power flow and short circuit calculation method of distribution network with inverter based DG is proposed according to the similarities between equivalent models of inverter based DG in normal and fault operation conditions. Finally, the proposed method is verified in an example. It is convenient to use the integrated calculation method to calculate

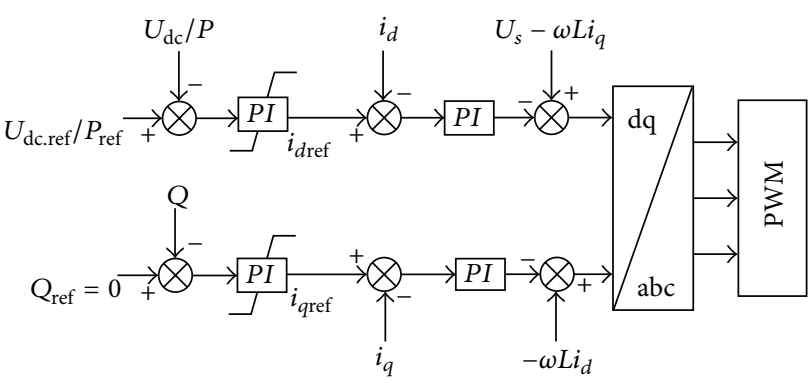

FIGURE 1: Control block of inverter.

power flow or short circuit current in practical engineering.

\section{Equivalent Model of Inverter Based DG}

The output characteristics of inverter based DG during normal and fault operation conditions of distribution network are associated with the control strategy of inverter. The dualloop control is usually adopted, which outer loop control targets are usually constant active and reactive power or constant DC voltage and reactive power. The output signals from outer loop are reference signals of inner ring, which mainly adopt $d-q$ decoupled control $[16,17]$. The control principle of inverter with current limiting function, which orients $d$-axis to grid voltage vector, is shown in Figure 1.

In Figure 1, $P_{\text {ref }}, Q_{\text {ref }}$, and $U_{\text {dc.ref }}$ are the references of active power, reactive power, and DC voltage, respectively. $P, Q$, and $U_{\mathrm{dc}}$ are the actual values of active power, reactive power, and DC voltage, respectively. $U_{S}$ is PCC voltage of DG. $i_{d}, i_{q}$ are the $d-q$ components of output current of inverter, respectively. $i_{d \text { ref }}, i_{q \text { ref }}$ are the references of $i_{d}$ and $i_{q}$, respectively. $\omega$ isangular frequency and $L$ is filter inductance of AC side.

Thus, whatever the outer loop control target is, the inner loop control for inverter is current control invariably. According to the instantaneous power theory, the active and reactive power exchange between DG and grid can be calculated with [18]

$$
\begin{gathered}
P=U_{S} i_{d}, \\
Q=U_{S} i_{q} .
\end{gathered}
$$

Inverter based DG usually works under constant power control in normal operation condition. In this case, it can be equivalent to $P Q$ bus because the active and reactive power output from DG is known. However, the distribution network with heavy loads will reduce network voltage. At this moment, the $d$-axis and $q$-axis current will increase according to (1), and the output current maximum $I_{\max }$ from DG is usually less than 1.2 1.5 times of its rated current according to its maximum allowable current threshold. After inverter enters into current limiting status, the output current magnitude of DG will be a constant. Along with the decreasing voltage, the output power from DG cannot follow the constant power control, which means that the DG cannot be equivalent to $P Q$ bus any more. In fault operation condition of distribution 
network, the DG may enter into the current limiting status in the same way easier due to the decreased PCC voltage caused by fault.

After inverter enters into current limiting status, the magnitude of output current from DG is $I_{\max }$. In unit power factor control mode, the phase difference $\theta$ between output current and PCC voltage of DG is $0^{\circ}$, while in nonunit power factor control mode, the power factor is given normally, which means the phase difference $\theta$ is known. Accordingly, DG can be equivalent to $I \theta$ bus at current limiting status. $I$ is $I_{\max }$ and $\theta$ is the phase difference between the output current and PCC voltage of DG. Hence, the equivalent model of inverter based DG should be changed from $P Q$ bus to $I \theta$ bus when inverter enters the current limiting status in both power flow and short circuit calculation.

\section{The Difference between Short Circuit Calculation and Power Flow Calculation}

Through the analysis of equivalent models for inverter based DG during normal and fault conditions of distribution network, it can be seen that the similarity between them is that DG should be modeled as $P Q$ and $I \theta$ buses, respectively, before and after inverter entering current limiting status. Hence, an integrated power flow and short circuit calculation method is proposed in this paper, which takes the idea of power flow calculation into short circuit calculation. This method can calculate both power flow and short circuit current of distribution network. Since the power flow calculation method of this paper is only applicable to symmetric system, the integrated calculation method based on it is only applicable to symmetrical fault calculation. In addition, short circuit calculation itself is different from power flow calculation whereby the following two problems have to be solved in short circuit calculation.

3.1. The Modification of Bus Admittance Matrix for Distribution Network. In three-phase short circuit calculation, the node admittance matrix should be modified by following three reasons.

(1) Loads Model. The loads of distribution network are omitted in three-phase symmetrical short circuit calculation usually. If the impacts of loads on short circuit current have to be taken into consideration, the loads model should be changed from constant power model to constant impedance model. This is because the calculation would not converge due to the unbalanced power flows in downstream distribution network of fault point. Then, the bus admittance matrix of distribution network should be modified after the loads model is changed. The short circuit calculation in this paper has ignored the loads of distribution network.

(2) Grounding Resistance. Since fault point only exists in fault condition of distribution network, the circuit structures of distribution network during normal and fault conditions are different. In fault condition, the fault happens in somewhere of distribution network, which means that a grounded branch is connecting to the fault point, as shown in Figure 2. $z_{f}$ in
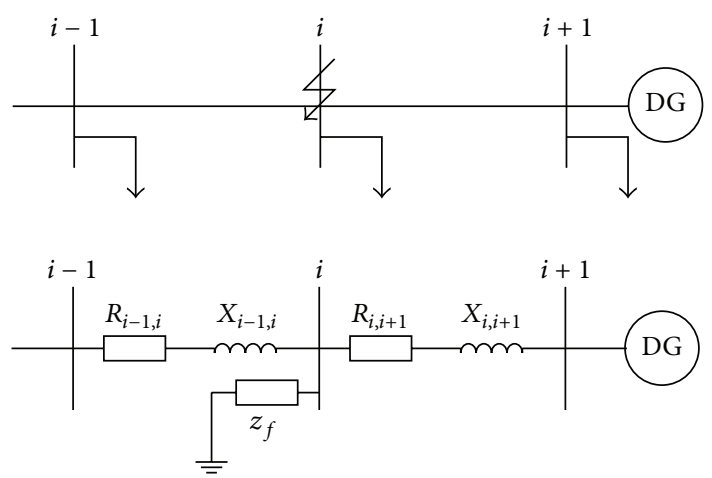

FIGURE 2: Equivalent circuit of distribution network in fault condition.

Figure 2 is grounding resistance $\left(z_{f}=0\right.$ under ideal metallic short circuit).

From Figure 2 it can be seen that there is a new added grounding admittance at fault point $i$. So that, in short circuit calculation, the self-admittance of fault point $i$ needs to be modified according to the following equation:

$$
Y_{i i . f}=Y_{i i . n}+\frac{1}{z_{f}},
$$

where $Y_{\text {ii.f }}$ and $Y_{i i . n}$ are the self-admittance of bus $i$ of postfault and prefault distribution network, respectively.

(3) DG in Downstream of the Fault Point. In distribution network, the fault may occur in any position of line. If there are multiple DGs in same distribution network, the DGs can be divided into two categories which are DGs in upstream of fault point and DGs in downstream of fault point. In past short circuit calculation method of distribution network with DG, the short circuit current contribution from DG in downstream of fault point has not been taken into account generally [19]. This is because the power capacity of DG in distribution network is small. Hence, the DG in downstream of fault point would be disconnected from distribution network under low voltage protection. Although the DG still connects to distribution network, the short circuit current from DG is small enough, thereby only the short circuit current contribution from DG in upstream of fault point is usually considered.

However, short circuit current from the DGs in downstream of fault point is larger, if more DGs are installed in that area. In addition, DG is requested to keep grid-connection for a period of time during a fault. Therefore, the short circuit current contribution from DGs in downstream of fault point should not be ignored.

For example, the DG in Figure 2 is located in downstream of fault point, and an isolated circuit is formed between fault point and DG. At this moment, the output current from DG fails to follow its control target, since the output characteristics of DG are only associated with line impedance characteristics. To simplify the calculation, the DG in downstream of fault point should be regarded as current source and modeled as $I \theta$ bus with $I=I_{\max }, \theta=0^{\circ}$. Then the 
parameters of the lines between DG and fault point should be updated from complex impedance to impedance modulus value $z\left(z=\sqrt{r^{2}+x^{2}}\right)$. And the bus admittance matrix of distribution network needs to be modified.

3.2. LVRT Fault Equivalent Model of DG. Both in normal and in fault operation conditions of distribution network, the equivalent model of inverter based DG mentioned above is analyzed based on the assumption of DG under constant power. However, in order to make full use of reactive power capacity of inverter when a fault takes place, the DG has usually had the ability of LVRT [20]. The fault equivalent model of DG with LVRT control is more complex because the reactive power outputs from DG and $\theta$ are variable.

It is important to note that the output characteristics of DG in downstream of fault point are only associated with line impedance characteristics, so that the LVRT model can only be applied to the DG in upstream of fault point. In order to take the short circuit current contribution from DG with LVRT control into consideration, it is necessary to analyze the LVRT equivalent model of inverter based DG.

Although the LVRT standards of different types of inverter based DG are different, the control idea of them is the same. When a fault occurs in distribution network, whether the DG adopts the LVRT control or not is decided by PCC voltage detected by control system. In LVRT control, the reactive current reference value of control system is adjusted according to the PCC voltage. Then the reactive power output from DG can be adjusted too. In this paper, the LVRT fault equivalent model of DG is described as follows.

(1) $U_{D G} \geq 0.9 p u$. In this situation, DG still maintains original constant power control, and the LVRT control cannot be switched on. The fault equivalent model is

$$
\begin{gathered}
P_{\mathrm{DG}}=P_{\mathrm{DG} 0}, \\
Q_{\mathrm{DG}}=Q_{\mathrm{DG} 0},
\end{gathered}
$$

where $U_{\mathrm{DG}}$ is the PCC voltage of DG, $P_{\mathrm{DG}}$ and $Q_{\mathrm{DG}}$ are the active power and reactive power output of $\mathrm{DG}$, respectively, and $P_{\mathrm{DG} 0}$ and $Q_{\mathrm{DG} 0}$ are the prefault active and reactive power output of DG.

(2) $0.4 p u<U_{D G}<0.9 p u$. In this situation, the PCC voltage of DG drops below 0.9 pu and DG starts to output reactive power current according to expression as follows:

$$
I_{q}=K\left(1-U_{\mathrm{DG}}\right) I_{N},
$$

where $I_{N}$ is the per unit rated current of inverter based DG. $K$ is the voltage adjustment coefficient of reactive power current output from DG. For example, $K=2$ means the voltage dropped $1 \%$ based on $1 \mathrm{pu}$; the reactive power current output from DG increased $2 \%$ of rated current. With an increase in reactive current output from DG, the DG may enter the current limiting status due to the maximum allowable current threshold. The judgment of current limiting status is the key to DG converting to $I \theta$ bus from $P Q$ bus. The current limiting status can be determined by the following equation:

$$
\sqrt{\left(\frac{P_{\mathrm{DG} 0}}{U_{\mathrm{DG}}}\right)^{2}+\left(K\left(1-U_{\mathrm{DG}}\right) I_{N}\right)^{2}}>I_{\max } .
$$

If (5) is not satisfied, the DG is not current limited. DG can still be equivalent to $P Q$ bus. The fault equivalent model is

$$
\begin{gathered}
P_{\mathrm{DG}}=P_{\mathrm{DG} 0}, \\
Q_{\mathrm{DG}}=I_{q} U_{\mathrm{DG}} .
\end{gathered}
$$

If (5) is satisfied, the DG is current limited. DG should be converted into $I \theta$ bus. The fault equivalent model is

$$
\begin{aligned}
I_{\mathrm{DG}} & =I_{\mathrm{max}} \\
\theta_{\mathrm{DG}} & =\arcsin \frac{I_{q}}{I_{\max }} .
\end{aligned}
$$

(3) $U_{D G} \leq 0.4 \mathrm{pu}$. In this situation, the PCC voltage of DG drops below $0.4 \mathrm{pu}$. In order to support voltage for distribution network adequately, DG should output reactive power current only. At this moment, the phase difference $\theta$ between the output current and PCC voltage of DG is $90^{\circ}$. The equivalent model of the DG can be modeled as $I \theta$ bus directly. The fault equivalent model is

$$
\begin{aligned}
& I_{\mathrm{DG}}=I_{\mathrm{max}}, \\
& \theta_{\mathrm{DG}}=90^{\circ} .
\end{aligned}
$$

From the LVRT equivalent model of DG, it can be seen that it is related to the PCC voltage of DG in current limiting status and noncurrent limiting status. Besides, whether the LVRT control is switched on or not, the fault equivalent models of DG are similar. Both the fault equivalent models can be modeled as $P Q$ and $I \theta$ buses, respectively, before and after inverter entering current limiting status. Therefore, the idea of power flow calculation can be used to short circuit calculation under LVRT control similarly.

\section{Integrated Calculation Method}

According to the analysis of equivalent model for inverter based DG during normal and fault operation conditions of distribution network, it can be seen that DG should have been converted into $I \theta$ bus in some operation scenarios. The integrated calculation method proposed in this paper uses the idea of power flow calculation to calculate short circuit current. However, the power flow calculation method in the past cannot calculate the power flow of distribution network with $I \theta$ bus directly. Hence, in this section, the conversion condition of bus type is analyzed and the power flow calculation method of distribution network with $I \theta$ bus is studied. 
4.1. Switching Condition of Bus Type. At the beginning of calculation, the present status of DG is unknown. Therefore, it should be assumed that when DG keeps the constant power control at present, the output current from DG is calculated according to results of past traditional power flow calculation method by (9) as follows:

$$
I_{\mathrm{DG} . i}=\left|\left(\frac{\widetilde{S}_{\mathrm{DG} . i}}{\dot{U}_{\mathrm{DG} . i}}\right)^{*}\right|,
$$

where $\widetilde{S}_{\mathrm{DG} . i}$ is the set value of output complex power from DG at bus $i$, under PQ control. $\dot{U}_{\mathrm{DG} . i}$ is the PCC voltage of DG. If $I_{\mathrm{DG} . i}>I_{\text {max. } i}$, the DG at bus $i$ enters into the current limiting status and should be switched to $I \theta . \theta$ depends on the output power factor of DG.

\subsection{Power Flow Calculation Method of Distribution Network} with $I \theta$ Bus. For $I \theta$ bus in distribution network, only its injected current $I$ and the phase difference $\theta$ between $I$ and PCC voltage are known. It is important to note that DG can be modeled as $Q I$ bus with $Q_{G i}=0$ while $\theta=0^{\circ}$ and can be modeled as $P I$ bus with $P_{G i}=0$ while $\theta= \pm 90^{\circ}$. But these two bus types can only be applied to the special cases that $\theta=0^{\circ}$ or $\theta= \pm 90^{\circ}$. In most cases, the current limited DG can be modeled as $I \theta$ bus, and the bus power equation of $I \theta$ bus can be expressed as

$$
\begin{aligned}
& U_{i} I_{i} \cos \theta_{i}-P_{L i}=U_{i} \sum_{j \in i} U_{j}\left(G_{i j} \cos \delta_{i j}+B_{i j} \sin \delta_{i j}\right), \\
& U_{i} I_{i} \sin \theta_{i}-Q_{L i}=U_{i} \sum_{j \in i} U_{j}\left(G_{i j} \sin \delta_{i j}-B_{i j} \cos \delta_{i j}\right),
\end{aligned}
$$

where $i \in I \theta$ bus, $U$ is the voltage amplitude, $\delta_{i j}\left(\delta_{i}-\delta_{j}\right)$ is the phase difference between bus $i$ and bus $j$, and $G_{i j}$ and $B_{i j}$ are the real and imaginary component of bus admittance matrix.

Let $n$ be the number of buses and $m$ be the number of $P Q$ buses; hence, the number of $I \theta$ buses is $n-m-1$. The simultaneous equations of power balance equations for $P Q$ and $I \theta$ buses are obtained as follows:

$$
\begin{aligned}
\Delta P_{1}(x)= & P_{G i}-P_{L i} \\
& -U_{i} \sum_{j \in i} U_{j}\left(G_{i j} \cos \delta_{i j}+B_{i j} \sin \delta_{i j}\right), \\
\Delta P_{2}(x)= & U_{i} I_{i} \cos \theta_{i}-P_{L i} \\
& -U_{i} \sum_{j \in i} U_{j}\left(G_{i j} \cos \delta_{i j}+B_{i j} \sin \delta_{i j}\right), \\
\Delta Q_{1}(x)= & Q_{G i}-Q_{L i} \\
& -U_{i} \sum_{j \in i} U_{j}\left(G_{i j} \sin \delta_{i j}-B_{i j} \cos \delta_{i j}\right), \\
\Delta Q_{2}(x)= & U_{i} I_{i} \sin \theta_{i}-Q_{L i} \\
& -U_{i} \sum_{j \in i} U_{j}\left(G_{i j} \sin \delta_{i j}-B_{i j} \cos \delta_{i j}\right),
\end{aligned}
$$

where in $\Delta P_{1}(x)$ and $\Delta Q_{1}(x), i \in 1,2, \ldots, m$ while in $\Delta P_{2}(x)$ and $\Delta Q_{2}(x) i \in m+1, m+2, \ldots, n-1$. In other words, subscript 1 represents $P Q$ bus and subscript 2 represents $I \theta$ bus. From (11), it can be seen that there are $2(n-1)$ equations, and the number of unknowns is also $2(n-1)$ including voltage amplitude and phase of each bus except slack bus. Therefore, the equations number is equal to the number of unknowns and the power flow calculation of distribution network with $I \theta$ bus can be solved. Equation (11) is a nonlinear equation which should be solved, so that the common Newton method is adopted in this paper.

The key problem to solving power flow by Newton method is the formation and computation of correction equations. According to solving process of Newton method, the correction equations are formed as follows:

$$
\begin{aligned}
{\left[\begin{array}{c}
\Delta P_{1} \\
\Delta P_{2} \\
\Delta Q_{1} \\
\Delta Q_{2}
\end{array}\right]=-\left[\begin{array}{llll}
H_{11} & H_{12} & N_{11} & N_{12} \\
H_{21} & H_{22} & N_{21} & N_{22} \\
M_{11} & M_{12} & L_{11} & L_{12} \\
M_{21} & M_{22} & L_{21} & L_{22}
\end{array}\right]\left[\begin{array}{c}
\Delta \delta_{1} \\
\Delta \delta_{2} \\
\frac{\Delta U_{1}}{U_{1}} \\
\frac{\Delta U_{2}}{U_{2}}
\end{array}\right], } \\
J=\left[\begin{array}{ll}
H & N \\
M & L
\end{array}\right] .
\end{aligned}
$$

The emphasis for solving the correction equations is the formation of Jacobian matrix $J$. Due to the emerging $I \theta$ bus in power flow calculation, the Jacobian matrix of past power flow calculation method cannot be used directly. From (12), it can be found that the Jacobian matrix is composed of four submatrixes which are $H, M, N, L$. According to the theory of Newton method, the expressions of diagonal elements in $N$ and $L$ are different from traditional power flow calculation method due to the introduction of $I \theta$ bus. The specific expressions are shown as follows.

Diagonal elements of $N$ are

$$
\begin{aligned}
N_{i i} & =\frac{\partial \Delta P_{i}}{\partial U_{i}} U_{i} \\
& =-U_{i} \sum_{\substack{j \in i \\
j \neq i}} U_{j}\left(G_{i j} \cos \delta_{i j}+B_{i j} \sin \delta_{i j}\right)-2 U_{i}^{2} G_{i i},
\end{aligned}
$$

$$
i \in 1,2, \ldots, m \text {, }
$$

$$
\begin{aligned}
N_{i i} & =\frac{\partial \Delta P_{i}}{\partial U_{i}} U_{i} \\
& =U_{i} I_{i} \cos \theta_{i}-U_{i} \sum_{\substack{j \in i \\
j \neq i}} U_{j}\left(G_{i j} \cos \delta_{i j}+B_{i j} \sin \delta_{i j}\right)
\end{aligned}
$$$$
-2 U_{i}^{2} G_{i i}, \quad i \in m+1, m+2, \ldots, n-1 .
$$ 
Diagonal elements of $L$ are

$$
\begin{aligned}
L_{i i}= & \frac{\partial \Delta Q_{i}}{\partial U_{i}} U_{i} \\
= & -U_{\substack{j \in i \\
j \neq i}} U_{j}\left(G_{i j} \sin \delta_{i j}-B_{i j} \cos \delta_{i j}\right)+2 U_{i}^{2} B_{i i}, \\
L_{i i}= & \frac{\partial \Delta Q_{i}}{\partial U_{i}} U_{i} \\
= & U_{i} I_{i} \sin \theta_{i}-U_{i} \sum_{\substack{j \in i \\
j \neq i}} U_{j}\left(G_{i j} \sin \delta_{i j}-B_{i j} \cos \delta_{i j}\right) \\
& +2 U_{i}^{2} B_{i i}, \quad i \in m+1, m+2, \ldots, n-1 .
\end{aligned}
$$

The initial values of voltage amplitude and phase of each bus should be given, respectively, after Jacobian matrix $J$ is formed, which are $\delta_{1}^{(0)}=\delta_{2}^{(0)}=0^{\circ}$ and $U_{1}^{(0)}=U_{2}^{(0)}=1$. According to the initial values, the error vectors $\Delta P_{1}^{(0)}, \Delta P_{2}^{(0)}$, $\Delta Q_{1}^{(0)}$, and $\Delta Q_{2}^{(0)}$ can be obtained. Then the next voltage amplitude and phase can be calculated according to (15) and (16) as follows:

$$
\begin{aligned}
& {\left[\begin{array}{c}
\Delta \delta_{1}^{(k)} \\
\Delta \delta_{2}^{(k)} \\
\frac{\Delta U_{1}^{(k)}}{U_{1}^{(k)}} \\
\left.\frac{\Delta U_{2}^{(k)}}{U_{2}^{(k)}}\right]
\end{array}\right]=-\left(J^{(k)}\right)^{-1} *\left[\begin{array}{c}
\Delta P_{1}^{(k)} \\
\Delta P_{2}^{(k)} \\
\Delta Q_{1}^{(k)} \\
\Delta Q_{2}^{(k)}
\end{array}\right], } \\
& \delta_{1}^{(k+1)}=\delta_{1}^{(k)}+\Delta \delta_{1}^{(k)}, \\
& \delta_{2}^{(k+1)}=\delta_{2}^{(k)}+\Delta \delta_{2}^{(k)} \\
& U_{1}^{(k+1)}=U_{1}^{(k)}+\Delta U_{1}^{(k)} \\
& U_{2}^{(k+1)}=U_{2}^{(k)}+\Delta U_{2}^{(k)} .
\end{aligned}
$$

Then substituting (16) into (15) to continue the iterative calculation until $\max \left|\Delta P_{1}^{(k)}, \Delta P_{2}^{(k)}\right|<\varepsilon$ and $\max \mid \Delta Q_{1}^{(k)}$, $\Delta Q_{2}^{(k)} \mid<\varepsilon$, the voltage amplitude and phase of each bus can be obtained. Moreover, the output power of current limited inverter based DG can be solved by

$$
\begin{array}{r}
P_{G i}=U_{i} \sum_{j \in i} U_{j}\left(G_{i j} \cos \delta_{i j}+B_{i j} \sin \delta_{i j}\right)+P_{L i}, \\
Q_{G i}=U_{i} \sum_{j \in i} U_{j}\left(G_{i j} \sin \delta_{i j}-B_{i j} \cos \delta_{i j}\right)+Q_{L i}, \\
i \in m+1, m+2, \ldots, n-1 .
\end{array}
$$

TABLE 1: PCC voltage and output current of DG.

\begin{tabular}{lcccc}
\hline DG no. & DG1 & DG2 & DG3 & DG4 \\
\hline Voltage/pu & 0.8612 & 0.8448 & 0.8573 & 0.8198 \\
Current/pu & 5.8058 & 5.9189 & 5.8320 & 6.0987 \\
\hline
\end{tabular}

4.3. Flowchart of Integrated Power Flow and Short Circuit Calculation Method. In this paper, there are two points which must be noted in the proposed integrated power flow and short circuit calculation method. First, there are some differences between short circuit and power flow calculation, so that the bus admittance matrix of distribution network and the corresponding fault equivalent model of DG should be modified for short circuit calculation. Second, if there are multiple DGs in same distribution network, the equivalent models of the rest of DGs may be affected by the changed voltage due to the equivalent model of one DG changed from $P Q$ bus to $I \theta$ bus. For this reason, the iterative process should be terminated until the equivalent model of each DG does not change any more. The specific flow chart of integrated power flow and short circuit calculation method is shown in Figure 3.

\section{Examples and Analysis}

In this paper, the numerical calculation is performed on a 33-bus distribution network, whose rated voltage is $10 \mathrm{kV}$. It is assumed that the same inverter based DGs are connected to buses 11, 17, 28, and 33, respectively, as shown in Figure 4, whose capacity is $0.5 \mathrm{MW}$. The per unit rated current of DG is 5 pu on the basis of the fact that base power and base voltage are $100 \mathrm{kVA}$ and $10 \mathrm{kV}$. It is assumed that the DGs work underrated condition with unit power factor mode and their output current maximum $I_{\max }$ is 1.2 times of rated current that is $6 \mathrm{pu}$. Bus 1 is slack bus whose voltage amplitude is $1 \mathrm{pu}$ and voltage phase angle is $0^{\circ}$. Then power flow calculation and short circuit calculation in this test system should be carried out by using the integrated calculation method proposed in this paper to verify its effectiveness.

5.1. Power Flow Calculation. In order to obtain current limiting DG, the loads in distribution network should be increased to 1.8 times of original value to obtain lower voltage in power flow calculation. According to calculation process, the calculation starts with DG equivalent to $P Q$ bus and the current limiting status of DG is estimated. The RMS of PCC voltage and output current of each DG are obtained as shown in Table 1. In this paper, all calculation results are expressed in per unit (pu) value.

From the calculation results in Table 1, it can be seen that only DG4 enters current limiting status. Accordingly, DG4 cannot maintain constant power output and should be equivalent to $I \theta$ bus. Then, according to the calculation process, the RMS of PCC voltage and output current of each DG are obtained by the power flow calculation method with $I \theta$ bus until the equivalent models of each DG are no longer changed. The calculation results are shown in Table 2 and the voltage profile of distribution network is shown in Figure 5. 


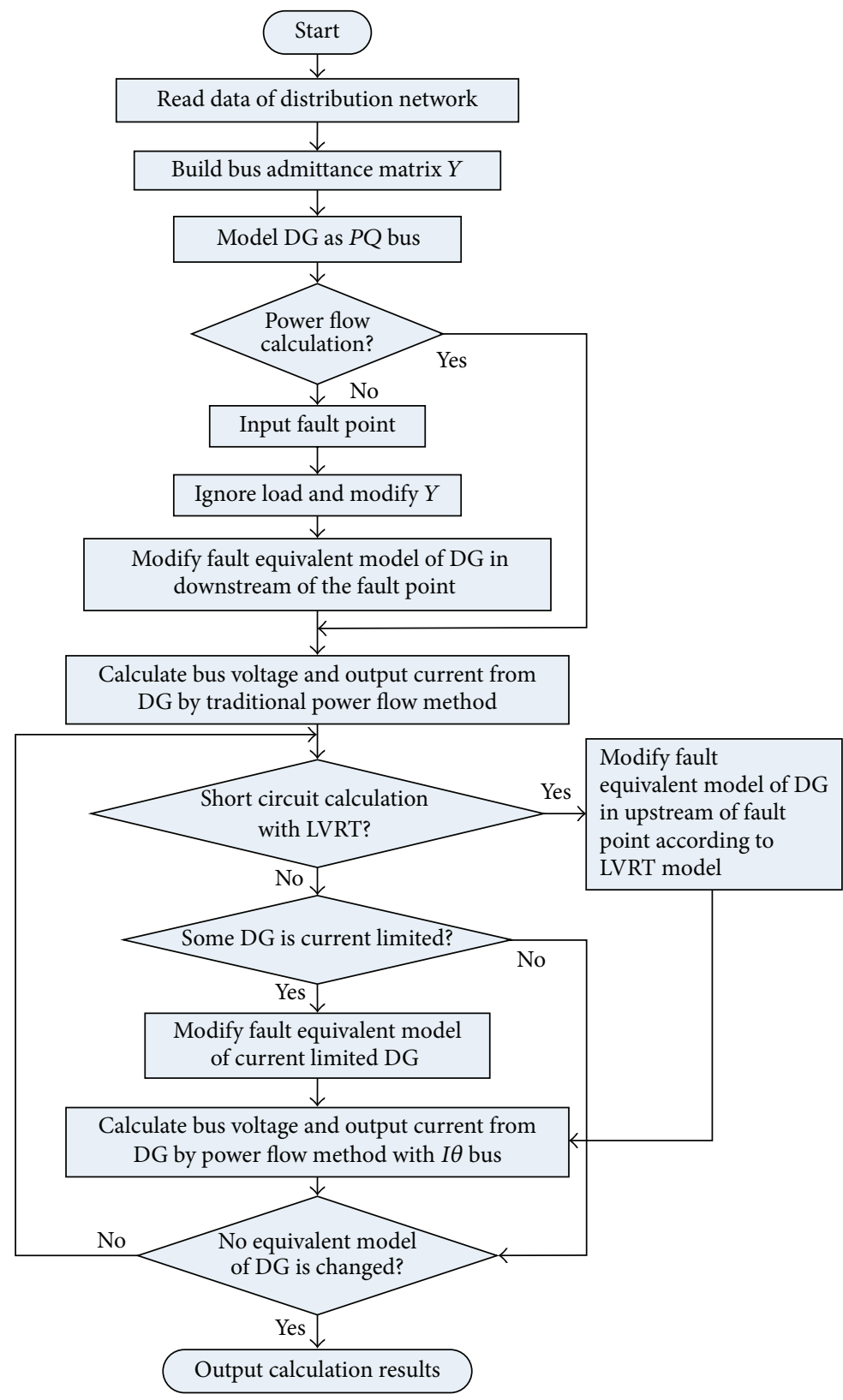

FIgURE 3: Flowchart of integrated calculation method.

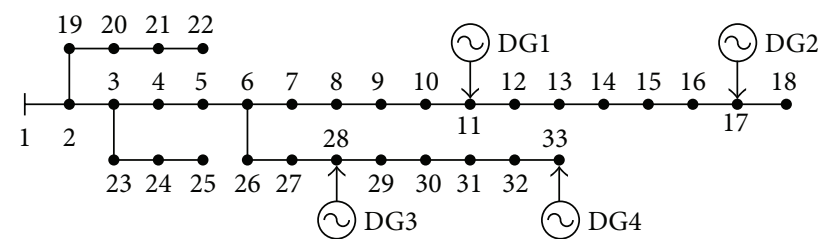

FIGURE 4: Structure chart of a 33-bus distribution system.

In order to verify if the power flow of distribution network with $I \theta$ bus can be correctly calculated by the integrated calculation method proposed in this paper, a method based on calculation results comparison between
TABLE 2: PCC voltage and output current of DG.

\begin{tabular}{lcccc}
\hline DG no. & DG1 & DG2 & DG3 & DG4 \\
\hline Voltage/pu & 0.8609 & 0.8445 & 0.8569 & 0.8191 \\
Current/pu & 5.8076 & 5.9207 & 5.8349 & 6.0000 \\
\hline
\end{tabular}

integrated calculation method and traditional calculation method is adopted. Because the $P Q$ values of DG under current limiting status are unknown, an inversion method is used. Concretely, the $P Q$ values of DG under current limiting status and the power flow of distribution network are calculated by the power flow method with $I \theta$ bus firstly. Then the traditional calculation method is used to calculate 


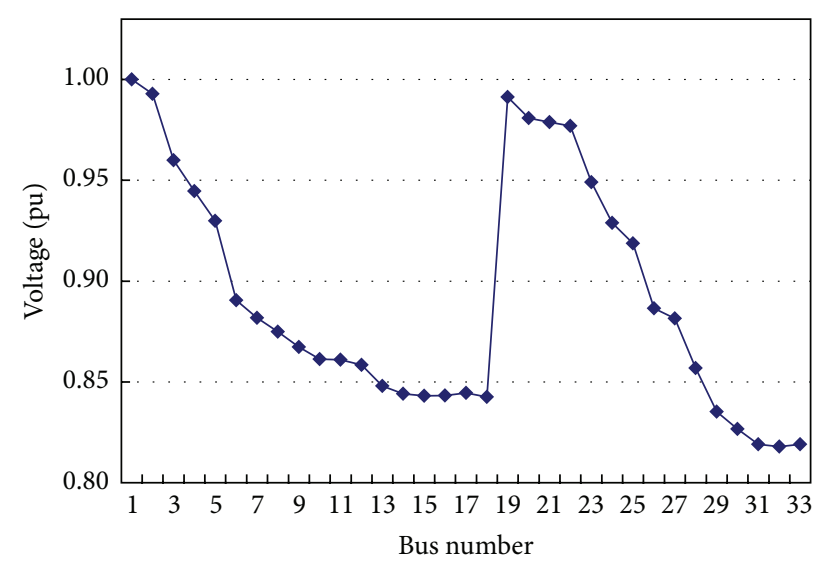

FIGURE 5: Voltage profile of distribution network.

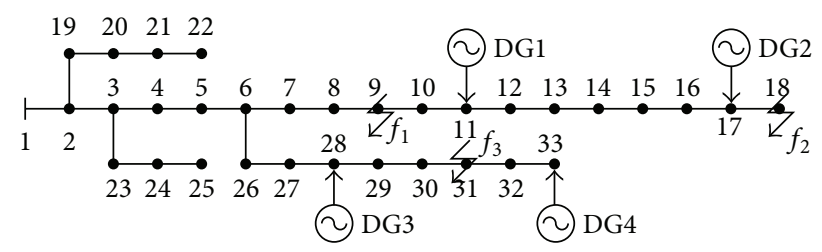

FIGURE 6: Structure chart of a 33-bus distribution system with fault points.

the power flow with the calculated $P Q$ values. The correctness of proposed integrated method can be verified if the power flows calculated by two methods are the same. In this case, the power flow of distribution network and active power output from current limited DG4 are calculated by integrated method at first. And the calculated active power output from DG4 is $4.9146 \mathrm{pu}$. Then the power flow is again calculated by traditional power flow calculation method with DG4 modeled as $P Q$ bus, whose active power is $P=4.9146 \mathrm{pu}$. Lastly, the maximum absolute value error of voltage of each bus calculated by two methods is $7.4884 e-6$. So that the proposed integrated method in this paper is correct.

5.2. Short Circuit Calculation for DG without LVRT Control. Similarly, the short circuit current is calculated by the integrated calculation method proposed in this paper in the same 33-bus distribution network. The three-phase grounding faults with grounding resistance are 0 , which are set on buses 9, 18, and 31, respectively, as shown in Figure 6. The fault positions set in this paper can be divided into the following four cases. All DGs in distribution network are in upstream of fault point (f2), some DGs are in downstream of fault point ( $\mathrm{fl}$ and $\mathrm{f} 3$ ), the fault occurs in trunk line ( $\mathrm{f} 1$ and $\mathrm{f} 2$ ), and the fault occurs in branch line (f3). According to the calculating process, the DG in upstream of fault point is equivalent to $P Q$ bus and the DG in downstream of fault point is equivalent to $I \theta$ bus at first calculation. The output current from DGs is obtained as shown in Table 3.

From the calculation results, it can be seen that the DG in distribution network is easy to enter current limiting status during faults. If the DG in upstream of the fault point
TABLE 3: Short circuit current output from each DG.

\begin{tabular}{lccc}
\hline \multirow{2}{*}{ Output short circuit current/pu } & \multicolumn{3}{c}{ Fault point } \\
& f1 & f2 & f3 \\
\hline DG1 & 6 & 8.3929 & 6.8759 \\
DG2 & 6 & 50.9485 & 6.5835 \\
DG3 & 9.2628 & 5.7244 & 11.7298 \\
DG4 & 8.8464 & 5.6192 & 6 \\
\hline
\end{tabular}

TABLE 4: Short circuit current comparison results between calculation and simulation.

\begin{tabular}{lccc}
\hline Short circuit current/pu & \multicolumn{3}{c}{ Fault point } \\
& f1 & f2 & f3 \\
\hline Calculation & 212.45 & 77.74 & 143.97 \\
Simulation & 210.79 & 77.54 & 143.98 \\
Error/100\% & $0.79 \%$ & $0.25 \%$ & $0.007 \%$ \\
\hline
\end{tabular}

TABLE 5: Part of bus voltage of distribution network.

\begin{tabular}{lccc}
\hline Bus voltage/pu & \multicolumn{3}{c}{ Fault point } \\
\hline 3 & $\mathrm{f} 1$ & $\mathrm{f} 2$ & $\mathrm{f} 3$ \\
6 & 0.8751 & 0.9626 & 0.9192 \\
9 & 0.5072 & 0.8499 & 0.6798 \\
11 & 0 & 0.6781 & 0.7020 \\
14 & 0.0181 & 0.5788 & 0.7166 \\
17 & 0.0371 & 0.3524 & 0.7305 \\
18 & 0.0603 & 0.0724 & 0.7461 \\
28 & 0.0603 & 0 & 0.7461 \\
31 & 0.5253 & 0.8673 & 0.4173 \\
33 & 0.5387 & 0.8801 & 0 \\
\hline
\end{tabular}

enters the current limiting status, its fault equivalent model should be converted into $I \theta$ bus. In this case, the output current from current limited DG is $I_{\max }$ and $\theta=0^{\circ}$ for the DG without LVRT control. Then the calculation process is continued until the equivalent models of each DG are no longer changed. The short circuit currents of $\mathrm{fl}, \mathrm{f} 2$, and f3 are calculated. Moreover, the simulation of this example is built in MATLAB/Simulink. The calculation results by integrated calculation method are compared with those by the simulation method in MATLAB/Simulink, with their results shown in Table 4.

From the data in Table 4, it can be seen that the results based on the integrated calculation method proposed in this paper are similar to the simulation results and the errors between them are reasonable, whereby the effectiveness and correctness of the proposed method are verified. Besides, the calculation results of the part of bus voltage of distribution network are shown in Table 5.

5.3. Short Circuit Calculation for DG with LVRT Control. In this case, the DG in upstream of fault point is equivalent to $P Q$ bus and the DG in downstream of fault point is equivalent to $I \theta$ bus similarly at first. The calculated current 
TABLE 6: Short circuit current comparison results between calculation and simulation.

\begin{tabular}{lccc}
\hline Short circuit current/pu & \multicolumn{3}{c}{ Fault point } \\
& f1 & f2 & f3 \\
\hline Calculation & 211 & 77.42 & 144.80 \\
Simulation & 211.28 & 77.54 & 145.69 \\
Error/100\% & $0.13 \%$ & $0.15 \%$ & $0.61 \%$ \\
\hline
\end{tabular}

TABLe 7: Part of bus voltage of distribution network.

\begin{tabular}{lccc}
\hline Bus voltage/pu & \multicolumn{3}{c}{ Fault point } \\
& $\mathrm{f} 1$ & $\mathrm{f} 2$ & $\mathrm{f} 3$ \\
\hline 3 & 0.8757 & 0.9630 & 0.9199 \\
6 & 0.5102 & 0.8519 & 0.6839 \\
9 & 0 & 0.6822 & 0.7172 \\
11 & 0.0182 & 0.5830 & 0.7320 \\
14 & 0.0371 & 0.3539 & 0.7479 \\
17 & 0.0603 & 0.0721 & 0.7689 \\
18 & 0.0603 & 0 & 0.7689 \\
28 & 0.5330 & 0.8698 & 0.4199 \\
31 & 0.5507 & 0.8829 & 0 \\
33 & 0.5573 & 0.8868 & 0.0068 \\
\hline
\end{tabular}

outputs from DGs are the same as those in Table 3. Then the current limiting status of DG in upstream of fault point is judged according to the calculation results. According to the LVRT equivalent model, the output current and the phase difference between output current and PCC voltage of DGs in current limiting status are calculated, and also, the output active and reactive power of DGs in noncurrent limiting status are calculated. Then the calculation process is continued until the equivalent models of each DG are no longer changed. In this paper, the short circuit currents of three kinds of fault are calculated according to the integrated calculation method. Moreover, the simulation of this example is built by MATLAB/Simulink. The calculation results by integrated calculation method are compared with those by the simulation method in MATLAB/Simulink, with their results shown in Table 6.

From the data in Table 6, it can be seen that the results based on the integrated calculation method are similar to the simulation results and the errors between them are reasonable. In addition, the calculation results of the part of bus voltage of distribution network are shown in Table 7 .

From the short circuit calculation results in Tables 5 and 7 , it can be seen that the bus voltages by short circuit calculation of DG with LVRT control are bigger than those without LVRT control. It is indicated that the LVRT control has had an increasing effect upon bus voltage, which reflects its advantage in the case of distribution network fault.

\section{Conclusion}

According to the theoretical analysis and simulation verification for the integrated power flow and short circuit calculation method of distribution network with inverter based DG, the following conclusions can be obtained:

(1) The equivalent models for inverter based DG during normal and fault conditions of distribution network are similar to each other. Both equivalent models can be modeled as $P Q$ and $I \theta$ buses, respectively, before and after inverter entering current limiting status. Therefore, the power flow and short circuit current can be calculated in the same way by an integrated power flow and short circuit calculation method.

(2) If distribution network has $I \theta$ bus, the expressions of elements related to $I \theta$ bus in Jacobian matrix being a part of correction equation for power flow calculation are different from those by traditional power flow calculation. The expressions should be reformed according to the power balance equations of $I \theta$ bus. Then power flow calculation by solving the correction equations can be completed.

(3) LVRT control is a conventional control of DG when a fault takes place in a distribution network. Hence, the LVRT equivalent model, which is more complicated than equivalent model in normal operation condition, should be analyzed.

(4) The distinction and similarity of power flow calculation and short circuit calculation should be attended for using the idea of power flow calculation to calculate short circuit current. And the adaptive complements for short circuit calculation are added in the process of integrated calculation method. The integrated power flow and short circuit calculation method proposed in this paper can be directly applied to practical engineering applications.

\section{Competing Interests}

The authors declare that there is no conflict of interests regarding the publication of this paper.

\section{Acknowledgments}

This work has been supported by the National Natural Science Foundation of China (51507139), the Key Discipline Special Foundation of Shaanxi Province, China (5X1301), and the Major Scientific and Technological Innovation Projects of Shaanxi Province, China (2015ZKC02-01).

\section{References}

[1] A. Khamis, H. Shareef, E. Bizkevelci, and T. Khatib, "A review of islanding detection techniques for renewable distributed generation systems," Renewable and Sustainable Energy Reviews, vol. 28, pp. 483-493, 2013.

[2] P. Paliwal, N. P. Patidar, and R. K. Nema, "Planning of grid integrated distributed generators: a review of technology, objectives and techniques," Renewable and Sustainable Energy Reviews, vol. 40, pp. 557-570, 2014.

[3] M. A. Haj-Ahmed and M. S. Illindala, "The influence of inverter-based DGs and their controllers on distribution network 
protection," in Proceedings of the IEEE Industry Applications Society Annual Meeting (IAS '13), pp. 1-9, October 2013.

[4] Y. Zhu and K. Tomsovic, "Adaptive power flow method for distribution systems with dispersed generation," IEEE Transactions on Power Delivery, vol. 17, no. 3, pp. 822-827, 2002.

[5] S. Li, "Power flow modeling to doubly-fed induction generators (DFIGs) under power regulation," IEEE Transactions on Power Systems, vol. 28, no. 3, pp. 3292-3301, 2013.

[6] Y. Ju, W. Wu, and B. Zhang, "Convergence problem in forward/backward sweep power flow method caused by nonpositive-sequence impedance of distributed generators and its solution," International Journal of Electrical Power \& Energy Systems, vol. 65, pp. 463-466, 2015.

[7] P. A. N. Garcia, J. L. R. Pereira, S. Carneiro, and V. M. Da Costa, "Three-phase power flow calculations using the current injection method," IEEE Transactions on Power Systems, vol. 15, no. 2, pp. 508-514, 2000.

[8] H. Shateri, M. Ghorbani, N. Eskandari, and A. H. MohammadKhani, "Load flow method for unbalanced distribution networks with Dispersed Generation units," in Proceedings of the 47th International Universities Power Engineering Conference (UPEC '12), pp. 1-7, September 2012.

[9] C. Wang and X. Sun, "An improved short circuit calculation method for distribution network with distributed generations," Automation of Electric Power Systems, vol. 36, no. 23, pp. 54-58, 2012.

[10] T. N. Boutsika and S. A. Papathanassiou, "Short-circuit calculations in networks with distributed generation," Electric Power Systems Research, vol. 78, no. 7, pp. 1181-1191, 2008.

[11] Y. Shan, T. Xiangqian, L. Jian et al., "Short-circuit current calculation of distribution network with distributed generation," Power System Technology, vol. 39, no. 7, pp. 1977-1982, 2015.

[12] N. Rajaei, M. H. Ahmed, M. M. Salama, and R. K. Varma, "Analysis of fault current contribution from inverter based distributed generation," in Proceedings of the IEEE PES General Meeting, Conference \& Exposition, pp. 1-5, National Harbor, Md, USA, July 2014.

[13] C. A. Plet, M. Graovac, T. C. Green, and R. Iravani, "Fault response of grid-connected inverter dominated networks," in Proceedings of the Power and Energy Society General Meeting, pp. 1-8, Minneapolis, Minn, USA, July 2010.

[14] N. K. Gouvalas, I. F. Gonos, and I. A. Stathopulos, "Impact study of short-circuit calculation methods on the design of a wind farm's grounding system," Renewable Energy, vol. 66, pp. 25-32, 2014.

[15] A. Uphues, K. Nötzold, R. Wegener, and S. Soter, "SOGI based grid fault detection for feeding asymmetrical reactive currents to fulfill LVRT requirements," in Proceedings of the IEEE AFRICON, pp. 1-5, Pointe-Aux-Piments, Mauritius, September 2013.

[16] W. ChenShan, L. Yan, and P. Ke, "Overview of typical control methods for grid-connected inverters of distributed generation," Proceedings of the CSU-EPSA, vol. 24, no. 2, pp. 12-20, 2012.

[17] R. Teodorescu, F. Blaabjerg, M. Liserre, and P. C. Loh, "Proportional-resonant controllers and filters for grid-connected voltage-source converters," IEE Proceedings: Electric Power Applications, vol. 153, no. 5, pp. 750-762, 2006.

[18] A. Ovalle, G. Ramos, S. Bacha, A. Hably, and A. Rumeau, "Decentralized control of voltage source converters in microgrids based on the application of instantaneous power theory,"
IEEE Transactions on Industrial Electronics, vol. 62, no. 2, pp. 1152-1162, 2015.

[19] I. Triştiu, C. Bulac, S. Costinaş, L. Toma, A. Mandiş, and T. Zăbavă, "A new and efficient algorithm for short-circuit calculation in distribution networks with distributed generation," in Proceedings of the 9th International Symposium on Advanced Topics in Electrical Engineering (ATEE '15), pp. 816-821, IEEE, Bucharest, Romania, May 2015.

[20] C.-T. Lee, C.-W. Hsu, and P.-T. Cheng, "A low-voltage ridethrough technique for grid-connected converters of distributed energy resources," IEEE Transactions on Industry Applications, vol. 47, no. 4, pp. 1821-1832, 2011. 


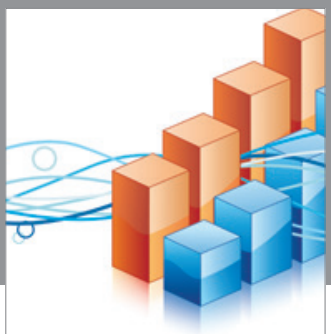

Advances in

Operations Research

vatem alat4

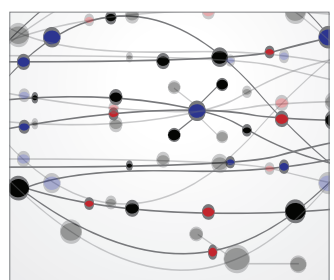

\section{The Scientific} World Journal
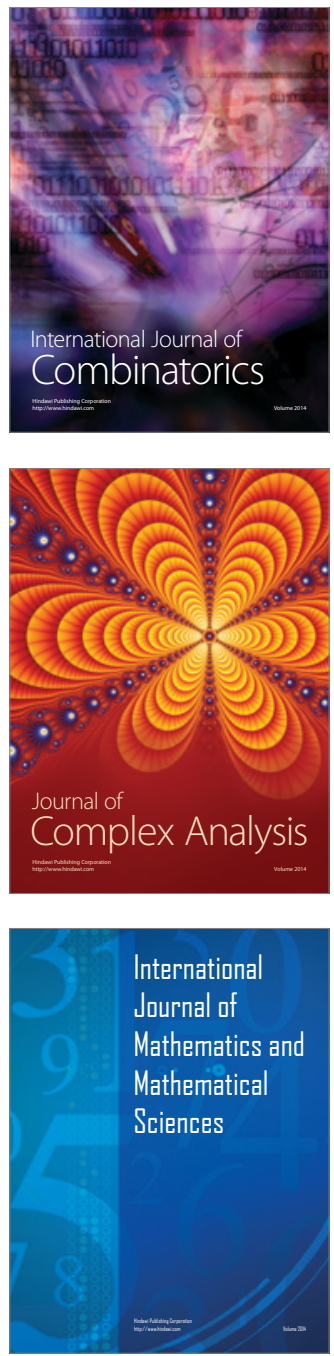
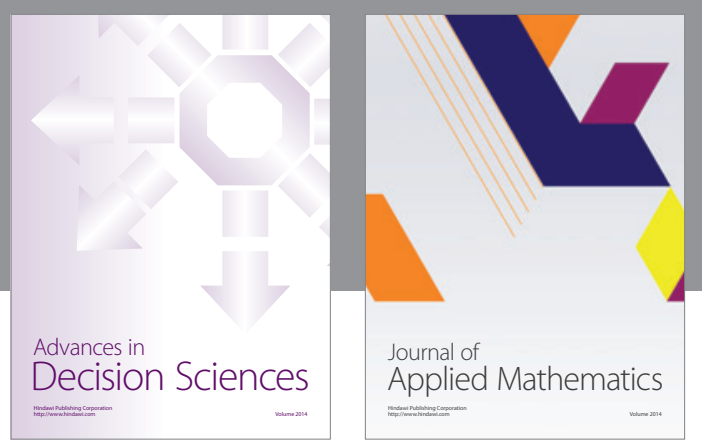

Algebra

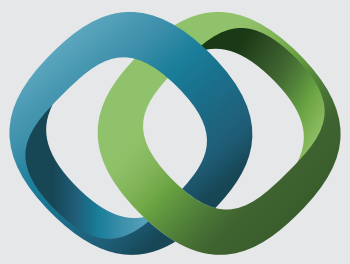

\section{Hindawi}

Submit your manuscripts at

http://www.hindawi.com
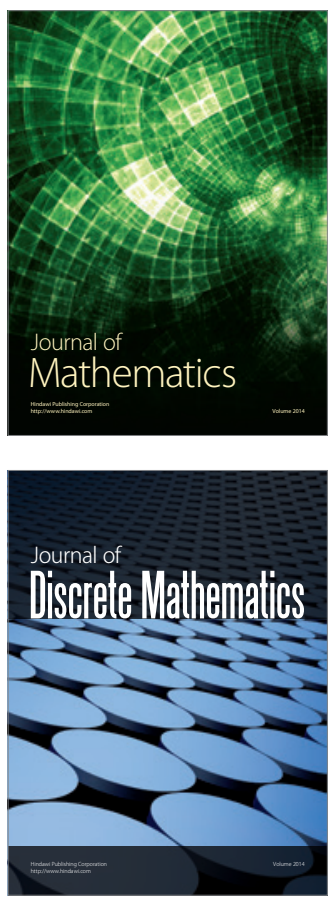

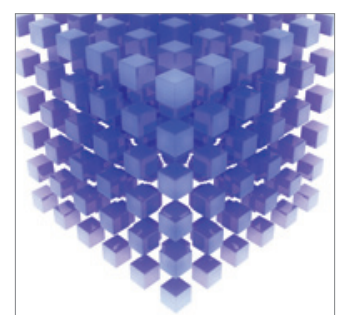

Mathematical Problems in Engineering
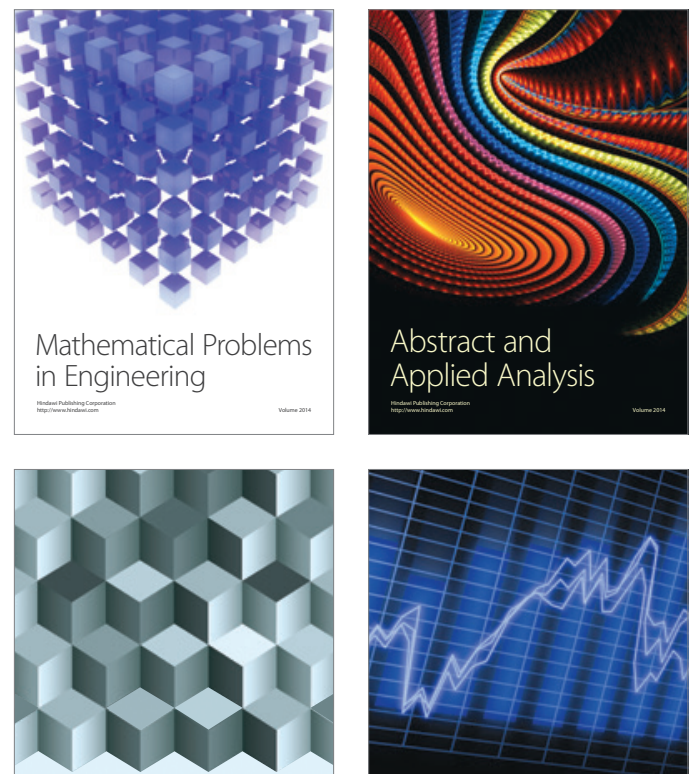

Journal of

Function Spaces

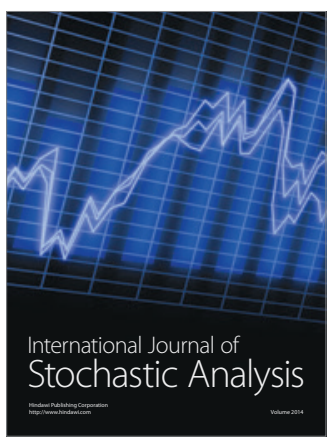

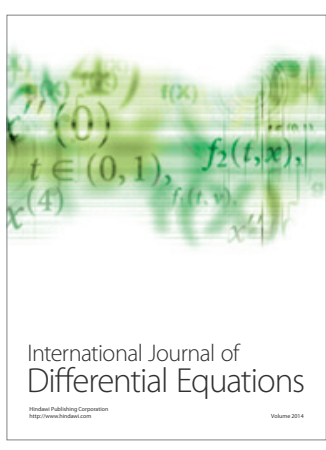
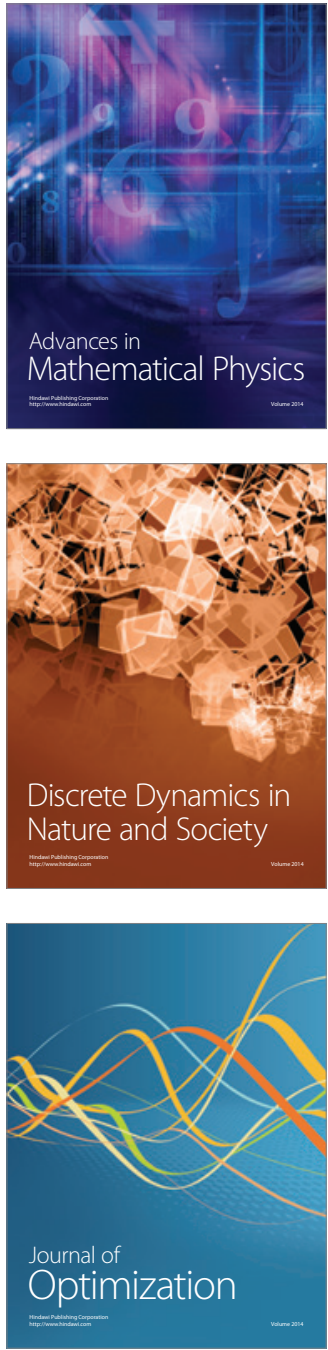